\title{
An Application of Human-Centered Multimedia System for Medical Implementation
}

\author{
Jesuk Ko* and Somkiat Kitjongthawonkul ${ }^{* *}$ \\ *Department of e-Business, Gwangju University \\ **School of Business \& Informatics, Australian Catholic University
}

\begin{abstract}
Multimedia interpretation describes a high level of abstraction of multimedia content like interrelations and coherencies. In this paper, we describe a multimedia interpretation component from human-centered perspective. In this context, we see the role of the multimedia interpretation component as a liaison between the psychological apparatuses and computer-based artifact. We emphasize this role of multimedia interpretation by outlining a mapping process of the psychological apparatuses and computer-based artifact. This mapping process needs to be transparent to its user so that effective human-centered systems can be achieved. This transparency will provide an immersive environment for the users and enable uninhibited interaction between the users and the artifact. Furthermore, we illustrate how the multimedia interpretation component can be utilized in the medical diagnosis and treatment support application.
\end{abstract}

Key Words : Human-Centered Systems, Multimedia Interpretation, Information Systems Development

\section{Introduction}

Over the past three decades, there has been a significant evolution of information systems - from being used as data processing mechanisms to more sophisticated decision making tools in organizations. This evolution has resulted in the emergence of technologies as well as methodologies for information systems development. Although there are a number of wide range of applications developed using this emergence of technologies and methodologies in areas such as database systems, management support systems, and intelligent systems, however, the usefulness of these applications is poor. The usefulness of applications or systems is not measured on how advance in technology be employed but is largely determined by how well the synergy between human and computers is [2].

Including the synergism between human and computers in part of information systems development is particularly important since the media equation theory [12] as well as literature [15] finds that the interaction between humans and their computers has a strong social component - humans treat their computers the way they treat other human beings.

According to previous study [4], the synergism between human and computers can be achieved by taking into account two joint aspects - leading to building effective humancentered systems: a) the way humans interact naturally with such systems to express emotion, mood, attitude and attention, and $b$ ) the human factors that pertain to multimedia data.

Since humans exhibit a social behavior when interacting with their computers [15] and the wide range of applications

Manuscript received Nov. 26, 2009; revised Dec. 5, 2009.

* Corresponding author: jko@gwangju.ac.kr (Jesuk Ko)

This research was conducted by research funds from Gwangju University in 2008, 2009. that use multimedia, and the multimedia content currently available, it implies that building effective systems requires not just only keeping human uses in mind but also a deep understanding of information at the semantic levels of multimedia content and interpretation of multimedia data [4]. However, most existing human-centered approaches focus on building effective human-centered systems from one aspect only (i.e. whether keeping human-centered issues in the loop or centering on user acceptance at the human-interface level). They do not seriously address building effective humancentered systems from system design or task level.

In this paper we firstly outline background of a humancentered systems development framework. This framework has taken into account the two aspects mentioned earlier. We then describe a multimedia interpretation component of the framework. We highlight the role play by this component in relation to building effective human-centered systems as well as how its principles can be realized in a real-world situation. Human-centered systems are problem solving driven, human activity-centered, and involve external and internal representation models of the domain.

The paper is organized as follows. Section 2 introduces the overall human-centered information systems development framework. From the software component perspective, the framework can be seen as consisting of three main components namely human activity-centered analysis component, problem solving ontology component, and multimedia interpretation component. More elaboration on human activity-centered analysis component can be found in [6] and that on the problem solving ontology component can be found in $[7,8]$. Section 3 describes the multimedia interpretation component of the framework in some detail. Section 4 illustrates the application of the multimedia interpretation component in a medical diagnostic system. Section 5 draws conclusion of this paper. 


\section{Background on Human-Centered Systems Development Framework}

The framework shown in Figure 1 is based on the guiding principles in various fields such as philosophy, cognitive science, psychology as well as work-oriented design and it is also in line with human-centered criteria as advocated by $[2,4$, 14]. More details of the framework can be found in $[6,8]$

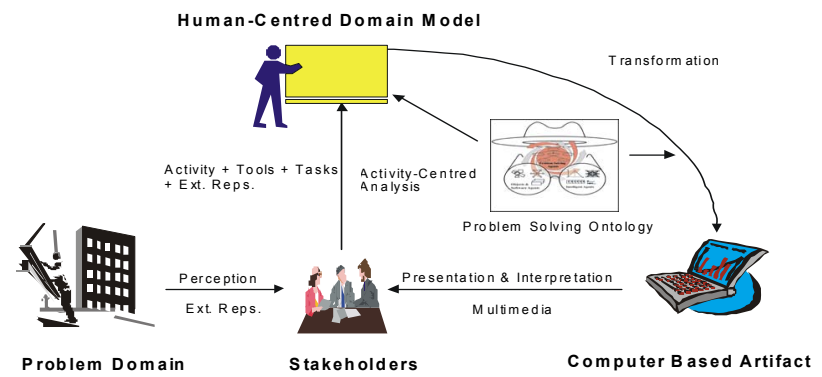

Fig. 1 Human-centered systems development framework

The information systems development framework can be realized as a software architecture consisting of three major components: the human activity-centered analysis component, the problem solving ontology component, and the multimedia interpretation component.

The human activity-centered analysis component helps to keep account of the physical, social and organizational reality on the external plane and the stakeholder's goals, tasks and incentives on the internal plane. The outcome of this analysis is a problem driven human-centered domain model which describes a high level of abstraction of various system components like activities, tasks, tools, inputs, products or outputs involved in the problem domain under consideration. This problem driven human-centered domain model not only incorporates some routine tasks but also accounts for breakdowns in the work situation. These breakdowns can also be seen as incentives for the stakeholders to use a computerbased artifact as a part of their workplace activity.

The problem solving ontology component is responsible for transformation of the human-centered domain model by systematizing and structuring the content and structure of the human-centered domain model using five information processing phases, namely, preprocessing, decomposition, control, decision, and postprocessing. The problem solving ontology component is derived from generalizing information processes and tasks as employed by people in large and complex systems rather than from any technological or cognitive stance. We see the role of problem-solving generalizations and routines grounded in experience as playing an important part in developing complex socio-technical systems. Thus, by accounting for the role separately, we can more effectively employ these generalizations to reach the outcomes of the human activity-centered analysis components that primarily focus on the existing problem setting or situation.

The multimedia interpretation component is responsible for making the psychological apparatuses mapped in the computerbased artifacts transparent to its users. It focuses on the humancomputer interaction in terms of how multimedia artifacts can be effectively used to model external representations, reduce the cognitive load of the computer-based artifacts on the stakeholders and enhance the perceptive aspects of problem solving. This can be seen in daily life where people use multimedia artifacts for communicating as well as accomplishing tasks in work situations

\section{Multimedia Interpretation}

Activity theory $[10,11]$ suggests that computer-based artifacts mediate work activity. The quality of mediation is based on social interaction between the user and the artifact. The quality of social interaction is determined by the extent to which the computer-based artifact is compatible with the psychological apparatuses or structures employed by the user in the work activity being modeled. Hence, the computer-based artifact can be seen as an extension of the psychological apparatuses of their users, which the users employ to facilitate their work activities. These psychological apparatuses influence the way the users perceive, use and learn the artifact. Thus, the interpretation of computer-based artifact should be based on among other aspects, the psychological scales and representing dimensions employed by the users on the psychological variables of interest rather than any physical variables used and computed by the computer-based artifacts to do the computation [13].

Multimedia interpretation is a description of media data at a high abstraction level, exposing interrelations and coherencies [9]. From human-centered perspective, the multimedia interpretation component is used to identify and analyze the psychological scales and representing dimensions used by direct stakeholders for gathering and providing information as well as psychological variables of interest used for interpretation of results. To make psychological apparatuses mapped in the computer-based artifact clear to the users, media artifacts such as graphics, text video and audio can be used to model the data perceptually so that the user's cognitive load may be reduced. This clarity will provide an immersive environment for the users and enable uninhibited interaction between the users and the artifact. The mapping process can be achieved through data content analysis and media selection.

\subsection{Data Content Analysis}

The data content analysis seeks to identify data for various tasks in a work activity context. The data content is defined according to its dimensionality, psychological scales, and representing dimensions as perceived by its users in the work activity and task context. The tasks in a work activity establish the context in which data is to be interpreted and used, which in turn define the human computer interaction points for data gathering as well as interpretation of computed results. 
The psychological scales and representing dimensions have been defined by the problem solving adapters of the problem solving ontology component. These psychological scales and representing dimensions are associated with data used for different tasks. In the context of a problem solving component, these psychological scales and representing dimensions are used to determine if perceptual reasoning techniques would be satisfactory for accomplishing the tasks. Nevertheless, these psychological scales and representing dimensions can also be used for data content analysis by the interpretation component.

The other data characteristics used for data content analysis include dimensionality, granularity, transience, urgency, and volume [1].

\subsection{Media Selection}

The aforementioned data characteristics influence the media, media substrata and media expression selection. Media specifies the type of medium used. Media substrate represents background to a simple exhibit. It establishes for the consumer, the physical or temporal relation and the semantic context, within which new information is presented to the information consumer or user, e.g. a piece of paper or screen - on which information may be drawn or presented or a grid - on which a maker might indicate the position of an entity. Media expression, on the other hand, determines the abstraction level of media.

Media expression can be classified on three levels [3] including elaborate level, representative level, and abstract level, as shown in Table 1. These terms are used to describe the degree of expressiveness of media type such as text, graphics, sound, and motion. For example, in order to display a customer order status, elaborate or representative text may be used as the media or medium, while a sliding scale may be used as a substrate with continuous granularity for indicating the progress of the order.

Table 1: Levels of media expression

\begin{tabular}{|c|c|c|c|}
\hline $\begin{array}{c}\text { Media } \\
\text { Type }\end{array}$ & $\begin{array}{c}\text { Elaborate } \\
\text { Media } \\
\text { Expression }\end{array}$ & $\begin{array}{c}\text { Representative } \\
\text { Media } \\
\text { Expression }\end{array}$ & $\begin{array}{c}\text { Abstract Media } \\
\text { Expression }\end{array}$ \\
\hline Text & $\begin{array}{c}\text { Fully expressed } \\
\text { written text }\end{array}$ & $\begin{array}{c}\text { Abbreviated } \\
\text { text, titles, } \\
\text { bulleted items }\end{array}$ & Shapes, Icons \\
\hline Graphics & $\begin{array}{c}\text { Fully expressed } \\
\text { photograph }\end{array}$ & $\begin{array}{c}\text { Abbreviated } \\
\text { blueprint, } \\
\text { layout }\end{array}$ & Graphic icon \\
\hline Sound & $\begin{array}{c}\text { Fully expressed } \\
\text { speech }\end{array}$ & $\begin{array}{c}\text { Abbreviated } \\
\text { tones }\end{array}$ & Sound effects \\
\hline Motion & $\begin{array}{c}\text { Fully expressed } \\
\text { film footage }\end{array}$ & $\begin{array}{c}\text { Abbreviated } \\
\text { animation, } \\
\text { news clips, } \\
\text { film preview }\end{array}$ & $\begin{array}{c}\text { Animated } \\
\text { model/icon }\end{array}$ \\
\hline
\end{tabular}

Selecting the medium and substrate based on a set of transformation rules have been suggested [1]. Some of the rules are defined here. For example, if the transience property is live, as a carrier, use a medium with the temporal endurance characteristic transient if the update rate is comparable to the lifetime of the carrier signal. If the data update rate is much longer, as a carrier, use a medium with the temporal endurance characteristic permanent. Regarding substrate, unless the information is already part of an existing exhibit, use neural substrate. If the transient property is dead, use a carrier/media with permanent temporal endurance. On the other hand, if the urgency property is urgent, then if the information is not yet part of a presentation instance, use a medium whose detectability has the value high either for substrate or carrier. If the information is already displayed as part of a presentation instance, use the present medium but switch one or more of its channels from fixed to the corresponding temporally varying state. Otherwise, if the property is routine, choose a medium with low default detectability and a channel with no temporal variance.

Finally, the selected media will be considered as to how it can be enhanced or be complemented by means of integrating it with other media at different levels of media expression or abstraction. For example, an elaborate selected media can be integrated with abstract or representative forms of other media to enhance understanding and develop a more immersive environment for the users. The level of media expression also facilitates mapping the media to different levels of problem solving. At higher levels of problem solving it is likely that abstract or representative levels of media expression will be mapped to data, whereas, at lower levels of abstraction elaborate levels of media expression are likely to be used.

\section{Medical Practitioner-Centered Multimedia Interface}

To illustrate how multimedia interpretation component of the framework can be effectively realized in a real-world situation, we outline its application in the medical diagnosis and treatment support system. Due to space limitation, only the multimedia interface for symptomatic data and gathering for Acute Otitis Media is covered here.

\subsection{Symptom Content Analysis}

The psychological variables employed by humans are invariably distinct from the physical variables used for computations by a computer-based artifact. For effective patient symptomatic data gathering in the drug prescription monitoring activity, it is useful to look at symptoms as psychological variables from a medical practitioner's perspective rather than as physical variables used for computation. In this section, we analyze the data characteristics of symptoms for Acute Otitis Media infection. We use the analysis to map the symptoms to various media artifacts.

The symptoms related to Acute Otitis Media are fever, sore ear, ear drum mild reddening or dullness, child screaming, child tugging ears, ear drum red or yellow and bulging, 
discharging ear, history of perforation, and has grommets. The Data characteristics such as dimensionality, psychological scale, representing dimension, granularity, transience and urgency have been used for the analysis. The psychological scale used is based on perceptive and cognitive or interpreted representations of the symptoms by the medical practitioners in the context of the drug prescription monitoring activity. The analysis of the symptoms has been done in the context of the drug prescription monitoring activity, which is briefly discussed as follows:

- Fever: is measured or determined on a single dimension of temperature. Medical practitioners measure the temperature in the range of 37 degrees Celsius to 40 degrees Celsius. Thus, the psychological scale information is on the interval scale, and the representing dimension is the position of mercury on this scale. The granularity of the fever symptom is considered as continuous (ranging from 37 degrees Celsius to 40 degrees Celsius). There is no urgency in terms of communicating the above information in the context of the drug prescription monitoring activity and thus it is classified as routine.

- Sore Ear: is measured on two dimensions, namely, location and color of the ear. The representing dimension of color is density which represents different color shades like skin (normal ear) color, pink (mild sore ear) color and red (sore ear) color in a continuous range. Here the red color represents higher severity than pink or skin color in terms of magnitude. Although, perceptually the soreness is indicated by the red color of the ear, which is indicative of the ordinal scale, the psychological scale used is ratio. This is because medical practitioners interpret the degree of soreness on a continuous scale, ranging from no soreness (zero) to yes ( 1 - indicated by the red color of the ear), to determine the strength of the treatment. The granularity is continuous and the urgency is routine.

- Child Screaming: consists of two dimensions, namely, location and density. These two dimensions are measured on the nominal and ratio scales, respectively. The location dimension relates to location of the screaming sound. The density dimension represents the screaming intensity, which is measured on a continuous scale of 0 (no) to 1 (yes). The property of a scream is transient.
- Ear Drum Red or Yellow and Bulging: consists of three dimensions, namely, location, color and shape. These three representing dimensions are perceived and interpreted on nominal, nominal and ratio scales, respectively. The location dimension relates to the ear drum location. The color of the eardrum is perceived and interpreted on the nominal scale based on the category property. However, the bulging shape or degree of bulge of the eardrum is perceived and interpreted on a ratio scale ranging from 0 to 1 with a continuous granularity.

- Discharging Ear: is measured on three dimensions, namely, location, color of the discharge and texture. These are also the representing dimensions of the symptom. The location dimension is based on the nominal scale, which includes the category property. The color dimension is based on the nominal scale and includes clear/transparent color discharge, yellow or green discharge. Finally, the texture dimension is based on the ordinal scale where the purulence of the discharge and the extent (magnitude) of discharge is determined. The granularity of this symptom is continuous and urgency is a routine.

- Has Grommets: represents two dimensions, namely, location and shape. These two dimensions are based on the nominal scale. The medical practitioner is looking for absence or presence of grommets only. Given the nominal scale on both the dimensions, the granularity is discrete.

The characteristics of these symptoms have been analyzed, based on characteristics such as dimension, psychological scale, representing dimension, granularity, and transience. These characteristics are most relevant to Acute Obits Media symptoms. Additional data characteristics such as volume and urgency have also been used in the analysis of treatment and other data. For example, the volume of symptoms is mostly represented by single facts and thus is singular, whereas, volume of treatment text, based on therapeutic guidelines, is considerable and requires elaborate text description.

\subsection{Media, Media Expression and Ornamentation Selection}

The characteristics of the symptoms outlined previously are used to select various media artifacts. Further, the modality or level of abstraction of various media is selected to facilitate complementation rather than duplication of media. In this section, we outline mapping of symptom characteristics to media types and the use of different levels of media expression.

Table 2: Media representations of upper respiratory

\begin{tabular}{|c|c|c|c|c|c|}
\hline Media/Data Characteristics & Fever & Sore Ear & Child Screaming & Discharging Ear & Has Grommets \\
\hline Media Type & $\begin{array}{c}\text { Text, image, sliding } \\
\text { scale }\end{array}$ & $\begin{array}{c}\text { Text, image, sliding } \\
\text { scale }\end{array}$ & $\begin{array}{c}\text { Text, image and } \\
\text { audio }\end{array}$ & Text image, sliding scale & $\begin{array}{c}\text { Text image } \\
\text { Check box }\end{array}$ \\
\hline Media Expression & Text - representative & Text - representative & Text - representative \\
Image - abstract & $\begin{array}{c}\text { Image - } \\
\text { representative } \\
\text { Sliding temperature } \\
\text { scale - elaborate }\end{array}$ & $\begin{array}{c}\text { Sliding color scale - } \\
\text { representative }\end{array}$ & $\begin{array}{c}\text { Audio - elaborate } \\
\text { Sliding scale - } \\
\text { representative }\end{array}$ & $\begin{array}{c}\text { Sliding texture scale - } \\
\text { representative }\end{array}$ & $\begin{array}{c}\text { Text - representative } \\
\text { Image - elaborate } \\
\text { Check box - elaborate } \\
\text { representative }\end{array}$ \\
\hline
\end{tabular}


- Fever: A combination of text, image icon and a temperature sliding scale has been used to represent fever. A sliding temperature scale with an interval range of 37 degrees to 40 degrees represents the single dimensionality and interval scale. The sliding scale in Figure 2 is used as a media substrate for determining patient's temperature. The scale pointer is the information carrier through which the actual physical value is recorded internally. The temperature sliding scale also represents continuous granularity of temperature. As shown in Table 2, the text, thermometer icon and temperature scale represent different levels of media expression, which complement each other. The thermometer icon is an abstract image icon of temperature and complements the temperature scale. The temperature sliding scale represents an elaborate level media expression of temperature and the word "fever" is a representative textual concept for temperature.

- Sore Ear: Text, image and a sliding color scale are three media types employed to represent the two dimensions of the sore ear symptom in Table 2 . The degree of soreness or density is represented using a sliding color scale. It is used as the media substrate for measuring the degree of soreness. The color interval ranges from normal ear to a red sore ear. The image of the red sore ear represents the location dimension as well as an elaborate level of media expression. The sliding color scale also gives a representative level of media expression for the degree of soreness. The red sore image also complements the sliding color scale representation.

- Ear Drum Red or Yellow and Bulging: is represented on three dimensions, namely, location, color and shape. The location dimension on a nominal scale is represented by the bulging eardrum image. The color dimension on the nominal scale is represented by the check box as well as the bulging ear drum image. The shape dimension on the ratio scale is represented using the bulge sliding scale ranging from a flat ear drum (indicating a physical value of 0 ) to bulging oval shaped ear drum (indicating a physical value of 1).

- Child Screaming: has been shown in Table 2 for its variation in terms of use of text image and audio media artifacts. The image and audio artifacts shown in Figure 2 are an elaborate expression of a child's scream in the two media types. Although they are at the same level, they tend to complement rather than duplicate each other. Further, unlike perceptually oriented sliding scales used for other symptoms, a no/yes sliding scale has been used for this symptom. The aural nature of this symptom restricted us somewhat in providing a more perceptually meaningful sliding scale.

- Discharging Ear: is represented using text, image, a texture based color sliding scale and a check box. The nominal scale of location dimension is represented using an elaborate level of the image artifact. The check box is used to confirm or negate presence or absence of the discharge. If the discharge is present the texture and color of the discharge is determined on a texture based color sliding scale. It may be noted that the sliding scale does not start with zero (0). The transparent or clear discharge on the left end of the sliding scale in Figure 2 represents a physical value of 1 whereas, the thick greenish discharge on the right end of the sliding scale represents a physical value of 5 . Further, the combining of color and texture dimensions into one representation in Figure 2 is based on the assumption that color and texture vary concurrently and are interpreted together (rather than in isolation) for the purpose of determining severity of symptom and strength of the treatment.

- Has Grommets: is represented using the image shown in Figure 2. The image is employed to represent the location and shape dimensions on the nominal scale. The check box is used to confirm or negate presence or absence of grommets.

The main purpose of this section has been to enhance the precision or quality of symptom data gathering. In this light, the multimedia representations shown in Figure 2 provide a richer medium for effective symptom data gathering than the paper-based form currently used in Drug Prescription Monitoring Activity. These representations have been based on the psychological scales and representing dimensions employed by medical practitioners for determining the diagnosis and treatment of upper respiratory infections. The multimedia representations are expected to assist in a clearer explanation and detection of the differences and inconsistencies in the treatments prescribed by different medical practitioners.

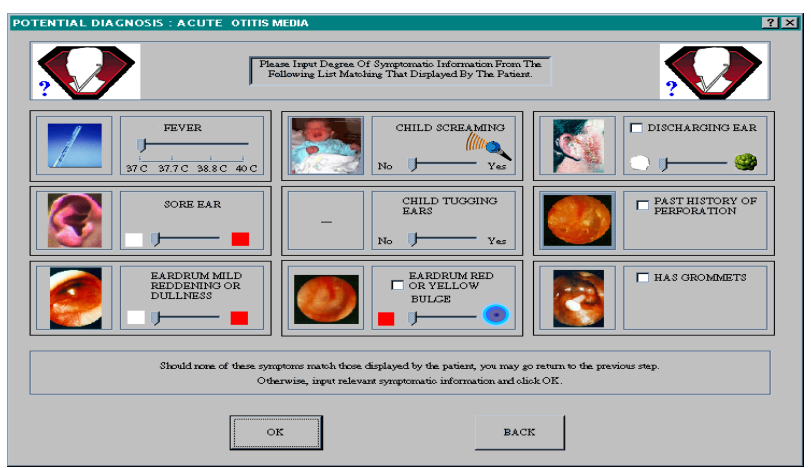

Fig. 2 Multimedia-based symptomatic data and gathering for Acute Otitis Media

In Figure 2, one can also notice two human faces with a question mark in the upper right and upper left-hand corners, respectively. These graphic objects are for the purpose of situating the medical practitioners in terms of information processing and patient diagnosis and treatment in the system. The screen in Figure 2 shows that the computerized system is trying to ascertain symptoms related to a potential diagnosis of Acute Otitis Media. Finally, for ornamentation, cyan color has been used as a back group color of the screen in Figure 2.

\section{Conclusion}

Many existing human-centered approaches are based on human factors engineering and usability engineering. These approaches primarily look at user acceptance issues at the human-computer interface level. We have adopted a more 
comprehensive approach, which includes, among other aspects, modeling human-centeredness not only at the interface but also at the underlying system design or task level. The humancentered information systems development framework adopted here has taken into account the two aspects of building effective human-centered system as advocated by [4] as well as grounded itself on human-centered criteria advocated by [4]. The human-centered information systems development framework consists of three main components: human activitycentered analysis component, problems solving ontology component, and multimedia interpretation component.

In this paper, we have limited our discussion on the multimedia interpretation component of the human-centered information systems development framework. The multimedia interpretation component is responsible for making seamless mapping of psychological apparatuses and computer-based artifact. In other words, it helps make the psychological apparatuses mapped in the computer-based artifact transparent to its users. The mapping process is achieved through using data content analysis and media selection. We also demonstrated the use of multimedia interpretation component in the medical diagnosis and treatment support application to develop practitioner-centered multimedia based patient data gathering forms.

We are currently working on the computational level architecture of the human-centered framework. The idea is to develop software layered architecture to facilitate a component based approach to system development.

\section{References}

[1] Y. Arens, E. H. Hovy, et al., On Knowledge Underlying Multimedia Presentations, Intelligent Multimedia Interface. T. Mark, AAAI Press, pp. 280-306, 1994.

[2] J. Flanagan and T. S. Huang, "Special Issue on HumanComputer Multimodal Interface," Proceedings of the IEEE, vol. 91, no. 9, 2003.

[3] R. Heller and B. Martin, "A Media Taxonomy," IEEE Multimedia, pp. 36-45, 1995.

[4] T. S. Huang, A. Jaimes, et al., Human-Centered Multimedia, Retrieved from http://research.microsoft.com/conferences/ ICME2007_files/TutorialDocs/5huang.pdf, October 2008.

[5] R. Khosla and S. Kitjongthawonkul, "A Human-Centered Agent-Based Architecture for Electronic Brokerage," International Journal of Soft Computing, vol. 5, no. 6, pp. 405411, 2001.

[6] S. Kitjongthawonkul, "Information Systems Development: A Human-Centered Approach," IADIS International Conference Information Systems 2008, Algarve, Portugal, 2008.

[7] S. Kitjongthawonkul and R. Khosla, "Task-Based Ontology for Information Systems," Proceedings of the 2000 Americas Conference on Information Systems (AMCIS 2000), Long Beach, California, USA, 2000.
[8] J. Ko and S. Kitjongthawonkul, "Task-Based Ontology of Problem Solving Adapters for Developing Intelligent Systems," International Journal of Fuzzy Logic and Intelligent Systems, vol. 4, no. 3, pp. 353-360, 2004.

[9] R. Moller and B. Neumann, Ontology-Based Reasoning Techniques for Multimedia Interpretation and Retrieval, Semantic Multimedia and Ontologies: Theories and Applications, P. M. Hobson and Y. Kompatsiaris, Springer Science+Business Media, 2008.

[10]B. A. Nadi, A Small Matter of Programming: Perspective on End User Computing, Cambridge, MIT Press, 1993.

[11]B. A. Nadi, Studying Context: A Comparison of Activity Theory, Situated Action Models, and Distributed Cognition. Context and Consciousness, MIT Press, pp. 69-102, 1996.

[12]C. Nass and B. Reeves, The Media Equation, New York, Cambridge University Press, 1996.

[13]D. A. Norman, The Psychology of Everyday Things, New York, Basic Books, 1988.

[14]D. A. Norman and S. W. Draper, User Centered System Design: New Perspectives on Human-Computer Interaction, Hillsdale, New Jersey, Lawrence Erlbaum Associates, Inc., 1986.

[15]H. Schaumburg, "Computers as Tools or as Social Actor?: The Users's Perspective on Anthropomorphic Agents," Journal of Coperative Information Systems, vol. 10, no. 1-2, pp. 217-234, 2001.

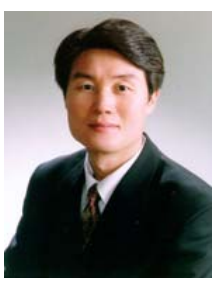

\section{Jesuk Ko}

He received the M.S. degree in Industrial Engineering from Pohang University of Science and Technology, Korea, in 1992, and the Ph.D. degree in Systems Engineering from Royal Melbourne University of Technology, Australia, in 1996. He is currently an Associate Professor in the Department of e-Business, Gwangju University. His research interests include expert systems, AI applications in manufacturing systems, and enterprise reengineering such as ERP, SCM, CRM, KM, etc.

\section{Somkiat Kitjongthawonkul}

He received the M.S. degree in Computer Science from Royal Melbourne University of Technology, Australia, in 1992, and the Ph.D. degree in Computer Engineering from La Trobe University, Australia, in 2002. He is currently a Lecturer in the School of Business and Informatics, Australian Catholic University. His research interests include business information systems, ubiquitous computing applications, multi-agent and distributed systems, EAI. 